\title{
Early onset high grade dysplasia in ulcerative colitis
}

\author{
D. Clements, V. Suarez, H. Thompson, J. Alexander-Williams and R.N. Allan
}

Gastroenterology Unit and Department of Histopathology, General Hospital, Steelhouse Lane, Birmingham B4 $6 N H, U K$.

\begin{abstract}
Summary: We report a patient with ulcerative colitis who underwent colectomy when aged 25 years for multifocal high grade dysplasia which had developed within 2 years of diagnosis, and only $5 \frac{1}{2}$ years after his first symptoms. The implications of this case for the management of ulcerative colitis are discussed.
\end{abstract}

\section{Introduction}

There is considerable interest in the development of dysplasia, and the risk of carcinoma in ulcerative colitis (UC). ${ }^{1}$ Regular surveillance has been advocated for patients with extensive colitis after 10 years from the onset of symptoms as most of the large bowel cancers occur after this stage and are associated with dysplasia in adjacent or distal mucosa. ${ }^{2-4}$ This surveillance usually involves annual colonoscopy with multiple biopsies to detect dysplasia. Although the dysplasia-carcinoma sequence has not been proved, colectomy is recommended by some when there is high grade dysplasia on two occasions. ${ }^{3,4}$

\section{Case report}

A 22 year old male presented in July 1982 with an extensive necrotic leg ulcer and ischaemic toes. For the previous 18 months he had had diarrhoea with occasional bleeding. Sigmoidoscopy, good quality double contrast barium enema and stool culture were normal; rectal biopsy was not performed. He was treated with antibiotics, and steroids as there was a vasculitic component to his leg ulcer. The ulcer subsequently required a skin graft and several gangrenous toes were amputated. His diarrhoea improved.

In February 1985 his diarrhoea recurred. Sigmoidoscopy then showed proctitis which was confirmed on rectal biopsy and associated with low grade dysplasia. A repeat barium enema examination demonstrated total colitis.

In August 1985 another rectal biopsy showed no evidence of dysplasia but in April 1986 flexible

Correspondence: D. Clements, M.R.C.P.

Accepted: 25 June 1987 sigmoidoscopy showed raised mucosal plaques and biopsies again showed low grade dysplasia. By July 1986 there was a severe total colitis on colonoscopy with multiple raised lesions and biopsies were taken. In these there were several areas of high grade dysplasia. ${ }^{3}$ When these widespread and severe changes were confirmed on repeat biopsy he underwent colectomy.

Blocks were taken at $3 \mathrm{~cm}$ intervals from the colon and from any raised or irregular areas of mucosa. There was a total colitis and multiple foci of both low and high grade dysplasia (Figure 1) were observed throughout the colon in both raised and flat mucosa. Carcinoma was not identified.

\section{Discussion}

This patient developed high grade dysplasia at an early age, and remarkably quickly after the onset of symptoms. Low grade dysplasia was present on his first rectal biopsy only 4 years after the initial onset of diarrhoea and only $2 \frac{1}{2}$ years after barium enema and sigmoidoscopy had been normal. Subsequent biopsies $5 \frac{1}{2}$ years after onset of symptoms revealed high grade dysplasia in both colon and rectum.

The concept that the colorectal cancer risk in UC is not significantly increased until 10 years after onset of symptoms may be misleading because some surveys of colorectal cancer complicating UC specifically excluded early cases of cancer. ${ }^{5}$ This eliminates referral bias from patients with UC and cancer at presentation, but exclusion has been based on the assumption that the colitis must have been longstanding, although asymptomatic, to be associated with cancer.

Establishing the precise date of onset of colitis is often difficult and is based on the duration of 


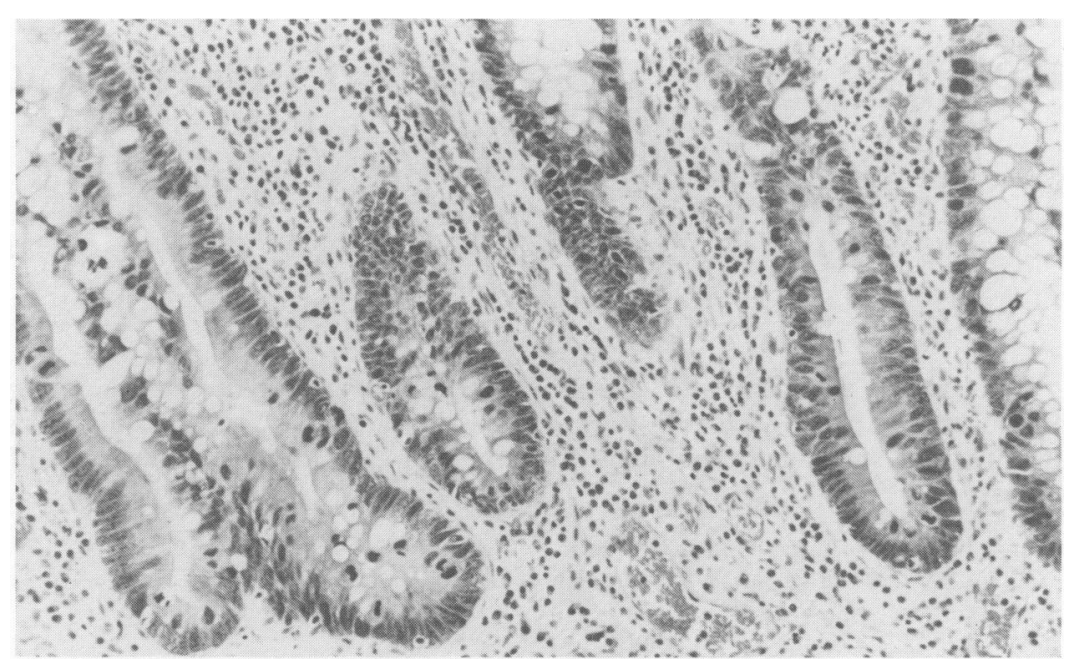

Figure 1 Epithelium showing high grade dysplasia. (Magnification $\times 125$ )

symptoms. In this case dysplasia was first identified only 3 years after developing diarrhoea and barium enema and sigmoidoscopy were normal less than 4 years prior to colectomy for high grade multifocal dysplasia.

This case demonstrates that UC of less than 10 years may develop high grade dysplasia and its detection would have been delayed in a surveillance programme which began only after 10 years of colitis. The present recommended surveillance programmes will therefore be inadequate and start too late for some patients. More accurate methods of dating the onset of colitis are needed before the relationship between dysplasia, cancer and the duration of colitis can be defined.

\section{References}

1. Editorial. Colorectal carcinoma in ulcerative colitis. Lancet 1986, ii: 197-198.

2. Lennard-Jones, J.E., Morson, B.C., Ritchie, J.K. \& Williams, C.B. Cancer surveillance in ulcerative colitis. Lancet 1983, ii: 149-153.

3. Riddell, R.H., Goldman, H., Ransohoff, D.F. et al. Dysplasia in inflammatory bowel disease: standardized classificiation with provisional clinical qualifications. Hum Pathol 1983, 11: 931-968.

4. Riddell, R.H. Dysplasia and cancer in ulcerative colitis: a soluble problem? Scand J Gastroenterol 1984, 14: 137-149.

5. Prior, P., Gyde, S.N., Macartney, J.C., Thompson, H., Waterhouse, J.A.H. \& Allan, R.N. Cancer morbidity in ulcerative colitis. Gut 1982, 23: 490-497. 